\title{
Hexagonal and Ring Packing Method for Solving Circle Packing Problem using Modified Heuristics
}

\author{
Apoorva Shastri, Saaloni Sharma, Aniket Nargundkar
}

\begin{abstract}
A heuristic technique is a perspective used for problem solving, which employs a problem specific domain knowledge which might not be optimal, but sufficient for the immediate goals. The most common heuristic is trial and error, employed everywhere and anywhere. One such application is Circle Packing Problem (CPP), which is difficult to solve as it is a NP problem which is of high theoretical and practical value. In this paper we consider uniform packing of unit circles (diameter 2) in an enclosing circle. To make this study possible a class of heuristic global optimization algorithm, the Energy Paving Algorithm and a generated Monte Carlo method were studied. The heuristics applied in this study are - ring and hexagonal. The computational effectiveness of our study has been validated against these sets of algorithms. One of the applied heuristics, Hexagon packing, being a 'natural' geometry showed better packing efficiency than existing literature. Our second heuristic shows, higher efficiency in terms of CPU run time but underutilized available outer area. In this study, the circles are packed in $2 D$ which is a simplified version of the various problems existing in nature as container loading, sensor network layout which has been studied by mathematicians, computer scientists and operation researchers.
\end{abstract}

Keywords: Circle Packing Problem, Novel Heuristics, Ring Packing, Hexagonal Packing.

\section{INTRODUCTION}

The circle packing problem is an arrangement of circles, in an optimized enclosed bounding circle, called the enclosing circle without any overlap. There are two approaches to find a dense solution of non over lapping circles. Place $\mathrm{N}$ over lapping circles of unit diameter into smallest possible circle of diameter $\mathrm{D}$. $\mathrm{N}$ equal circles to find the greatest diameter of the packed circles.Mostly, all the circles are packed mutually tangent to each other for high packing density. Lagrange, in 1773 proved that the highest density arrangement of circles in an unbounded Euclidean plane has centers of the circle arranged in a hexagonal lattice (honeycomb), where each side is surrounded by 6 other circles. These problems have been proved by mathematicians but the computer scientists are

Revised Manuscript Received on October 30, 2019.

* Correspondence Author

Apoorva Shastri*, ${ }^{1}$ Lovely Professional University, Phagwara, Punjab 144411 India, ${ }^{2}$ Symbiosis Institute of Technology, Symbiosis International (Deemed University), Lavale, Pune 412115 India.

Saaloni Sharma, ${ }^{3}$ DLF Centre,Bank of America ,Sansad Marg ,New Delhi 110001.

Aniket Nargundkar, ${ }^{2}$ Symbiosis Institute of Technology, Symbiosis International (Deemed University), Lavale, Pune 412115 India.

(C) The Authors. Published by Blue Eyes Intelligence Engineering and Sciences Publication (BEIESP). This is an open access article under the CC BY-NC-ND license (http://creativecommons.org/licenses/by-nc-nd/4.0/) trying to develop the most efficient heuristic. Circle packing can be done in both uniform packing and non-uniform packing.

The tragedy of commons, is when the problem that has no 'technical' solution, it requires a fundamental extension of morality [5]. Heuristic is a technique designed for solving a problem quicker than the classical method, or to find an approximate solution when the exact solutions fail. The objective of a heuristic is to solve the problem at hand as quickly as possible, which may not be the best possible solution but it can be computed in a reasonable time frame. Heuristics can be used alone or in conjunction with the already available algorithms to yield better seed values. In computer science, heuristics are the only possible solution for the NP problems with complex optimization properties.

The main criteria to check if the tradeoff of a heuristic is good are:

1. Optimality: is the solution found best amongst all the available.

2. Completeness: can the heuristic find all the possible solutions.

3. Accuracy and Precision: the error bar of the solution should be as minimum as possible.

4. Execution time: they should converge significantly faster than the classical methods.

Liu et al. considered the problem with given n objects and a container, each with given shape and size. The objective of problem is to pack the objects into the container avoiding overlap. The paper used ELP algorithm [1]. Wong et al. presented that the packing problems are combinatorial optimization with the aim of allocating multiple objects in a containment region without overlap. The target is to maximize the occupied space, minimizing wastage [2].

The paper is a study about developing a hybrid approach, combining grid approximation and genetic algorithm, to pack a set of irregular objects onto a stock sheet to improve the usability of the stock sheet [3]. $\mathrm{Li}$ investigated the applications of circle packing in the field of medicine. The paper employs the Monte Carlo method to study a constrained optimization problem- packing hard spheres with unequal radii (r2 > r1) into a 3D bounded region and discuss its connection with the Gamma Knife radio surgery treatment planning [4]. Smith A.P have used higher-dimensional viewpoint for problem analysis of quasicrystals that pack spheres [10]. George A.J et.al. applied heuristic methods on fitting circles of different sizes into a rectangle [6]. Kubach T. et.al. have applied greedy algorithms strip packing problem (SPP) and Knapsack Problem (KP) [7][8].Vassilios E et. al. demonstrated the effect of a polynomial-time cooling schedule on the computational performance of the algorithm [9]. 
In this paper modified heuristic approach is applied to achieve efficient circle packing of unit circles.

The paper is organized as follows: Section II in details describes the problem formulation. It includes the importance of the problems, mathematical formulations and the details about the methods solving the problems. Section III describes the methodology solving the CPP problem. It is followed by the discussion on the results and comparison in section IV. The conclusions and a comment on future direction are provided at the end.

\section{PROBLEM FORMULATION}

The problem formulation for CPP is taken from [1] as given below:

- $\quad \mathrm{n}$ circles $\mathrm{C}_{\mathrm{i}}$, with radius $\mathrm{r}_{\mathrm{i}}, \mathrm{i} \in N=\{1 \ldots n\}$

- central point of a larger circle containing circle $\mathrm{c}_{0}$ with the unknown radius $r_{0}$

- given circle should not extend beyond the outside larger containing circle, $\mathrm{c}_{0}$

$$
\sqrt{x_{i}^{2}+y_{i}^{2}} \leq r_{0}-r_{i}, i \in N
$$

- $\quad$ no two circles, should overlap each other placed in the given circle

$$
\sqrt{\left(x_{i}-x_{j}\right)^{2}+\left(y_{i}-y_{j}\right)^{2}} \geq r_{i}+r_{j}, i \neq j \in N
$$

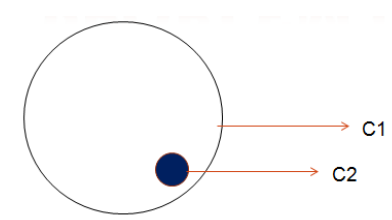

Fig.1 - Problem illustration

\section{METHODOLOGY}

\section{a. Hexagonal Packing -}

Hexagons are called the 'natural' shape. The geometrical properties of a Nobel hexagon can be derived using the Pythagoras Theorem. Let us call one edge $x$, as shown in the figure below. $1^{2}+x^{2}=2^{2}$. It then follows that $1+x^{2}=4, x^{2}=3$, and thus $x=\sqrt{ } 3$.

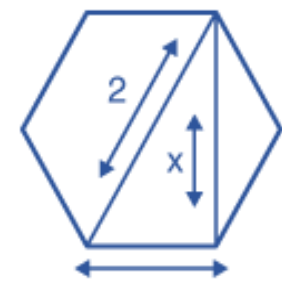

Fig.2 -Ratios of Nobel Hexagon

The intuitive analysis helps us to draw the following conclusions:

The apothem of a regular hexagon is equal to half the square root of 3.

The "height" to "width" of a regular hexagon is equal to $\sqrt{ } 3 / 2$. The minimum diameter of either a hexagon or a triangle is $\sqrt{3} / 2$ its maximum diameter. The point to ponder on is the recurrence of the value $\sqrt{3}$ throughout the hexagonal and triangular geometry discussion.
These hexagonal lattices can be stacked in a 2D plane infinitely, so the packing density is:

$$
\eta_{h}=\frac{\pi}{2 \sqrt{3}} \approx 0.9069
$$

For the most efficient space enclosure, each cell tends towards circularity. Also, each cell has neighboring cells pressing up against each other, which results in a quasi-hexagonal tiling of sorts.

\section{b. Ring Packing -}

In this approach the smaller circles are first packed along the circumference of the enclosing circle. Here we diameters of both the circles are given.

The angle subtended by one enclosed circle,

$$
d \theta_{1}=\sin ^{-1}\left(\frac{1}{R-r}\right)
$$

For a very small angle $\quad d l_{1}=2 r d \theta_{1}$

Now for the first ring, $\int d l_{1}=2 \pi(R-r)$

$\mathrm{N}$ represents the number of enclosed circles

$$
N_{l 1}=\frac{360^{\circ}}{2 \sin ^{-1}\left(\frac{1}{R-r}\right)}
$$

For the second loop, the circles sit in between the two circles, for efficient packing,

$$
\begin{aligned}
& \int d l_{2}=2 \pi(R-r-r \sqrt{3}) . \\
& d \theta_{2}=\sin ^{-1}\left(\frac{1}{R-r-r \sqrt{3}}\right)
\end{aligned}
$$

Number of circles in the second ring is given as

$$
N_{l 2}=\frac{360^{\circ}}{2 \sin ^{-1}\left(\frac{1}{R-r-r \sqrt{3}}\right)}
$$

This will continue until the center is reached. Finally, the summation of number of circles in each ring, gives us the following expression,

$$
N_{l n}=\frac{360^{\circ}}{2 \sin ^{-1}\left(\frac{1}{R-r(1+(n-1) \sqrt{3})}\right)}
$$

It is checked until $\int d l_{n}>2 \pi \mathrm{r}$.

When $\int d l_{n}=2 \pi \mathrm{r}$, then $N_{l n}=1$.

\section{RESULT AND DISCUSSION}

\section{HEXAGONAL PACKING}

The table below is a validation of all the 70 cases we simulated. The packing efficiency increases as and when the number of enclosed circle increases. 
Table- I Results Obtained For Hexagonal Packing

\begin{tabular}{|c|c|c|c|c|}
\hline \multicolumn{2}{|c|}{ BEST KNOWN [1] } & \multicolumn{3}{|c|}{ OUR RESULTS } \\
\hline $\begin{array}{r}\text { Number } \\
\text { of circles }(\mathrm{N})\end{array}$ & $\begin{array}{r}\text { CPU Run } \\
\text { time }\end{array}$ & $\begin{array}{l}\text { Number of } \\
\text { circles }(\mathrm{N})\end{array}$ & $\begin{array}{r}\text { CPU Run } \\
\text { time }\end{array}$ & $\begin{array}{l}\text { Efficiency } \\
\%\end{array}$ \\
\hline 31 & 45.15 & 31 & 65.88 & -45.91 \\
\hline 32 & 28.29 & 32 & 66.92 & -136.55 \\
\hline 33 & 78.35 & 33 & 65.8 & 16.02 \\
\hline 34 & 23.47 & 34 & 68.11 & -190.20 \\
\hline 35 & 29.38 & 35 & 69.34 & -136.01 \\
\hline 36 & 35.42 & 36 & 68.2 & -92.55 \\
\hline 37 & 90.17 & 37 & 69.52 & 22.90 \\
\hline 38 & 37.43 & 38 & 68.16 & -82.10 \\
\hline 39 & 57.21 & 39 & 73.32 & -28.16 \\
\hline 40 & 67.47 & 40 & 73.28 & -8.61 \\
\hline 41 & 72.88 & 41 & 22.03 & 69.77 \\
\hline 42 & 28.21 & 42 & 75.71 & -168.38 \\
\hline 43 & 72.33 & 43 & 76.44 & -5.68 \\
\hline 44 & 140.11 & 44 & 75.01 & 46.46 \\
\hline 45 & 71.21 & 45 & 74.9 & -5.18 \\
\hline 46 & 60.5 & 46 & 75.01 & -23.98 \\
\hline 47 & 123.47 & 47 & 78 & 36.83 \\
\hline 48 & 90.55 & 48 & 74.65 & 17.56 \\
\hline 49 & 49.72 & 49 & 74.88 & -50.60 \\
\hline 50 & 58.35 & 50 & 78.87 & -35.17 \\
\hline 51 & 112.34 & 51 & 79.8 & 28.97 \\
\hline 52 & 98.41 & 52 & 80.74 & 17.96 \\
\hline 53 & 234.1 & 53 & 81.44 & 65.21 \\
\hline 54 & 98.22 & 54 & 80.06 & 18.49 \\
\hline 55 & 123.47 & 55 & 78.95 & 36.06 \\
\hline 56 & 576.42 & 56 & 87.46 & 84.83 \\
\hline 57 & 546.44 & 57 & 91.06 & 83.34 \\
\hline 58 & 789.2 & 58 & 86.76 & 89.01 \\
\hline 59 & 576.42 & 59 & 87.39 & 84.84 \\
\hline 60 & 179.33 & 60 & 86.66 & 51.68 \\
\hline 61 & 432.55 & 61 & 90.17 & 79.15 \\
\hline 62 & 1457.78 & 62 & 85.88 & 94.11 \\
\hline 63 & 952.71 & 63 & 85.19 & 91.06 \\
\hline 64 & 2356.58 & 64 & 86.42 & 96.33 \\
\hline
\end{tabular}

Here, negative sign of efficiency indicates the underutilization of available outer circle space. As shown is Table I, as the number of circles increases, efficiency of occupying the available space of outer circle increases and resulted in only positive efficiency values.

\begin{tabular}{|c|c|c|c|c|}
\hline 65 & 1892.77 & 65 & 90.23 & 95.23 \\
\hline 66 & 4013.42 & 66 & 89.79 & 97.76 \\
\hline 67 & 1449.34 & 67 & 90.73 & 93.74 \\
\hline 68 & 5123.13 & 68 & 94.59 & 98.15 \\
\hline 69 & 921.35 & 69 & 94.52 & 89.74 \\
\hline 70 & 3277.82 & 70 & 93.97 & 97.13 \\
\hline 71 & 3200.02 & 71 & 96.69 & 96.98 \\
\hline 72 & 1798.23 & 72 & 97.3 & 94.59 \\
\hline 73 & 1234.55 & 73 & 94.83 & 92.32 \\
\hline 74 & 1617.78 & 74 & 98.21 & 93.93 \\
\hline 75 & 2231.01 & 75 & 100.85 & 95.48 \\
\hline 76 & 1982.43 & 76 & 100.23 & 94.94 \\
\hline 77 & 452.44 & 77 & 99.72 & 77.96 \\
\hline 78 & 1442.41 & 78 & 99.38 & 93.11 \\
\hline 79 & 5676.32 & 79 & 104.03 & 98.17 \\
\hline 80 & 992.45 & 80 & 102.68 & 89.65 \\
\hline 81 & 2356.3 & 81 & 105.83 & 95.51 \\
\hline 82 & 1231.25 & 82 & 106.38 & 91.36 \\
\hline 83 & 7823.44 & 83 & 106.5 & 98.64 \\
\hline 84 & 3215.41 & 84 & 106.37 & 96.69 \\
\hline 85 & 2752.55 & 85 & 105.39 & 96.17 \\
\hline 86 & 5670.23 & 86 & 106.37 & 98.12 \\
\hline 87 & 7892.38 & 87 & 103.8 & 98.68 \\
\hline 88 & 6723.24 & 88 & 106.08 & 98.42 \\
\hline 89 & 6597.16 & 89 & 106.02 & 98.39 \\
\hline 90 & 7892.38 & 90 & 113.44 & 98.56 \\
\hline 91 & 8633.77 & 91 & 108.83 & 98.74 \\
\hline 92 & 12013.72 & 92 & 114.81 & 99.04 \\
\hline 93 & 14201.33 & 93 & 116.01 & 99.18 \\
\hline 94 & 7982.15 & 94 & 114.66 & 98.56 \\
\hline 95 & 8947.89 & 95 & 115.48 & 98.71 \\
\hline 96 & 9213.45 & 96 & 120.66 & 98.69 \\
\hline 97 & 3340.55 & 97 & 124.11 & 96.28 \\
\hline 98 & 3728.99 & 98 & 118.43 & 96.82 \\
\hline 99 & 11208.44 & 99 & 122.52 & 98.91 \\
\hline 100 & 13045.72 & 100 & 116.712 & 99.11 \\
\hline
\end{tabular}




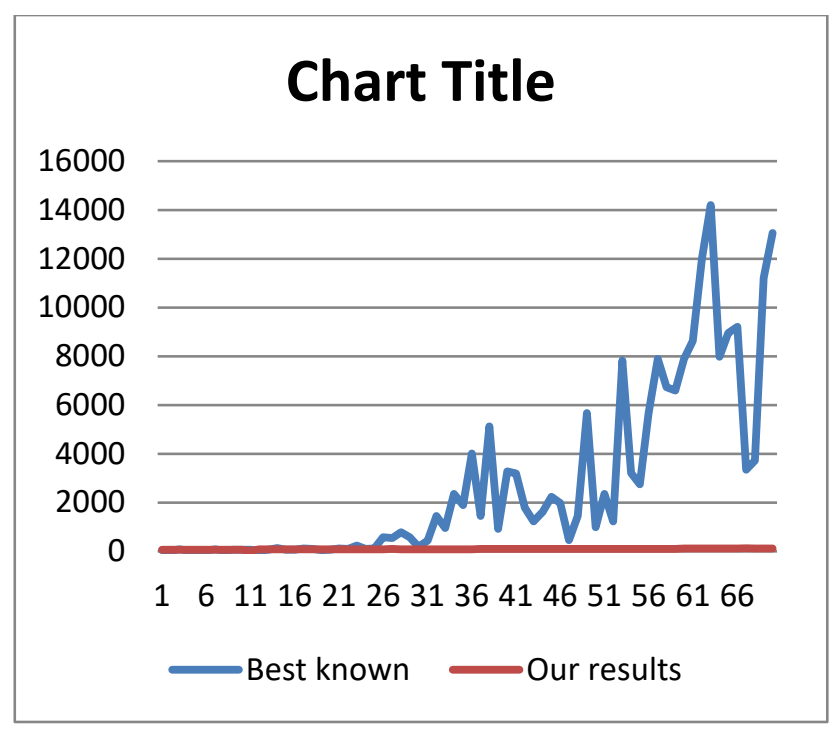

Fig.3 - CPU Run time hexagonal packing

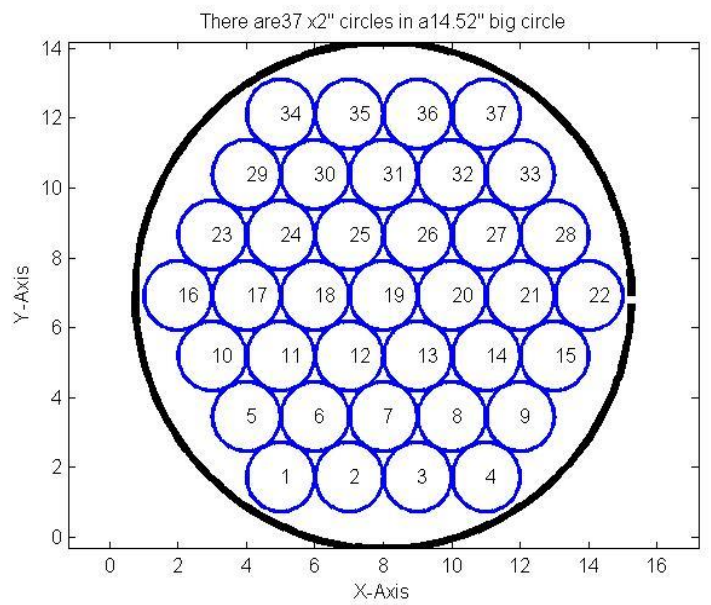

Fig. 4 - Simulation result for $\mathbf{N}=\mathbf{3 7}$

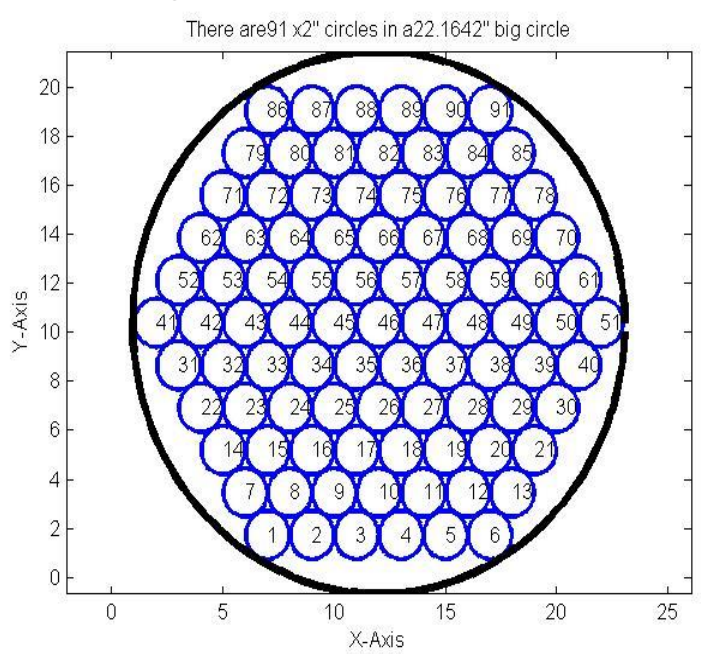

Fig.5 - Simulation result for $\mathbf{N}=\mathbf{9 1}$

Figure 3 to 5 shows the Matlab simulation results for ring and hexagonal packing respectively. Hexagonal packing shows better packing but underutilization of available outer circle area whereas in ring packing, overutilization is observed as shown in figures 2 and 4.

\section{RING PACKING}

The table below is a validation of all the 70 cases we simulated. The simulation time efficiency is minimum $99 \%$ for each case.

Table- II Results obtained for ring packing

\begin{tabular}{|c|c|c|c|c|}
\hline \multicolumn{2}{|c|}{ BEST KNOWN [1] } & \multicolumn{3}{|c|}{ OUR RESULTS } \\
\hline $\begin{array}{r}\text { Number } \\
\text { of circles (N) }\end{array}$ & $\begin{array}{r}\text { CPU Run } \\
\text { time }\end{array}$ & $\begin{array}{l}\text { Number of } \\
\text { circles (N) }\end{array}$ & $\begin{array}{r}\text { CPU Run } \\
\text { time }\end{array}$ & $\begin{array}{r}\text { Efficienc } \\
\text { y \% }\end{array}$ \\
\hline 31 & 45.15 & 31 & 0.12 & 99.73 \\
\hline 32 & 28.29 & 32 & 0.12 & 99.59 \\
\hline 33 & 78.35 & 33 & 0.18 & 99.77 \\
\hline 34 & 23.47 & 34 & 0.12 & 99.49 \\
\hline 35 & 29.38 & 35 & 0.12 & 99.59 \\
\hline 36 & 35.42 & 36 & 0.12 & 99.66 \\
\hline 37 & 90.17 & 37 & 0.13 & 99.86 \\
\hline 38 & 37.43 & 38 & 0.16 & 99.57 \\
\hline 39 & 57.21 & 39 & 0.11 & 99.80 \\
\hline 40 & 67.47 & 40 & 0.12 & 99.82 \\
\hline 41 & 72.88 & 41 & 0.16 & 99.79 \\
\hline 42 & 28.21 & 42 & 0.16 & 99.44 \\
\hline 43 & 72.33 & 43 & 0.15 & 99.79 \\
\hline 44 & 140.11 & 44 & 0.12 & 99.91 \\
\hline 45 & 71.21 & 45 & 0.13 & 99.82 \\
\hline 46 & 60.5 & 46 & 0.11 & 99.82 \\
\hline 47 & 123.47 & 47 & 0.14 & 99.88 \\
\hline 48 & 90.55 & 48 & 0.12 & 99.87 \\
\hline 49 & 49.72 & 49 & 0.11 & 99.77 \\
\hline 50 & 58.35 & 50 & 0.12 & 99.80 \\
\hline 51 & 112.34 & 51 & 0.14 & 99.88 \\
\hline 52 & 98.41 & 52 & 0.15 & 99.85 \\
\hline 53 & 234.1 & 53 & 0.14 & 99.94 \\
\hline 54 & 98.22 & 54 & 0.16 & 99.84 \\
\hline 55 & 123.47 & 55 & 0.16 & 99.87 \\
\hline 56 & 576.42 & 56 & 0.20 & 99.97 \\
\hline 57 & 546.44 & 57 & 0.15 & 99.97 \\
\hline 58 & 789.2 & 58 & 0.14 & 99.98 \\
\hline 59 & 576.42 & 59 & 0.14 & 99.98 \\
\hline 60 & 179.33 & 60 & 0.11 & 99.94 \\
\hline 61 & 432.55 & 61 & 0.11 & 99.97 \\
\hline 62 & 1457.78 & 62 & 0.18 & 99.99 \\
\hline 63 & 952.71 & 63 & 0.10 & 99.99 \\
\hline 64 & 2356.58 & 64 & 0.12 & 100.00 \\
\hline 65 & 1892.77 & 65 & 0.12 & 99.99 \\
\hline
\end{tabular}




\begin{tabular}{|c|c|c|c|c|}
\hline 66 & 4013.42 & 66 & 0.16 & 100.00 \\
\hline 67 & 1449.34 & 67 & 0.14 & 99.99 \\
\hline 68 & 5123.13 & 68 & 0.14 & 100.00 \\
\hline 69 & 921.35 & 69 & 0.14 & 99.99 \\
\hline 70 & 3277.82 & 70 & 0.14 & 100.00 \\
\hline 71 & 3200.02 & 71 & 0.14 & 100.00 \\
\hline 72 & 1798.23 & 72 & 0.16 & 99.99 \\
\hline 73 & 1234.55 & 73 & 0.16 & 99.99 \\
\hline 74 & 1617.78 & 74 & 0.16 & 99.99 \\
\hline 75 & 2231.01 & 75 & 0.14 & 99.99 \\
\hline 76 & 1982.43 & 76 & 0.21 & 99.99 \\
\hline 77 & 452.44 & 77 & 0.19 & 99.96 \\
\hline 78 & 1442.41 & 78 & 0.16 & 99.99 \\
\hline 79 & 5676.32 & 79 & 0.16 & 100.00 \\
\hline 80 & 992.45 & 80 & 0.16 & 99.98 \\
\hline 81 & 2356.3 & 81 & 0.18 & 99.99 \\
\hline 82 & 1231.25 & 82 & 0.19 & 99.98 \\
\hline 83 & 7823.44 & 83 & 0.19 & 100.00 \\
\hline 84 & 3215.41 & 84 & 0.19 & 99.99 \\
\hline 85 & 2752.55 & 85 & 0.19 & 99.99 \\
\hline 86 & 5670.23 & 86 & 0.18 & 100.00 \\
\hline 87 & 7892.38 & 87 & 0.19 & 100.00 \\
\hline 88 & 6723.24 & 88 & 0.20 & 100.00 \\
\hline 89 & 6597.16 & 89 & 0.23 & 100.00 \\
\hline 90 & 7892.38 & 90 & 0.20 & 100.00 \\
\hline 91 & 8633.77 & 91 & 0.19 & 100.00 \\
\hline 92 & 12013.72 & 92 & 0.16 & 100.00 \\
\hline 93 & 14201.33 & 93 & 0.17 & 100.00 \\
\hline 94 & 7982.15 & 94 & 0.21 & 100.00 \\
\hline 95 & 8947.89 & 95 & 0.20 & 100.00 \\
\hline 96 & 9213.45 & 96 & 0.25 & 100.00 \\
\hline 97 & 3340.55 & 97 & 0.24 & 99.99 \\
\hline 98 & 3728.99 & 98 & 0.20 & 99.99 \\
\hline 99 & 11208.44 & 99 & 0.20 & 100.00 \\
\hline 100 & 13045.72 & 100 & 0.28 & 100.00 \\
\hline
\end{tabular}

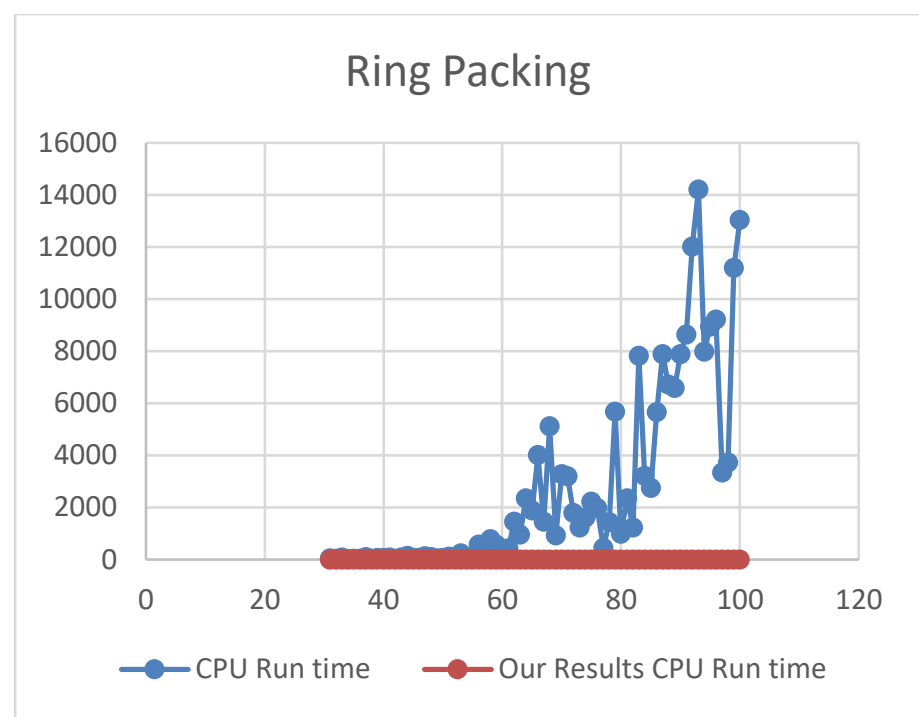

Fig.6 - CPU Run time ring packing

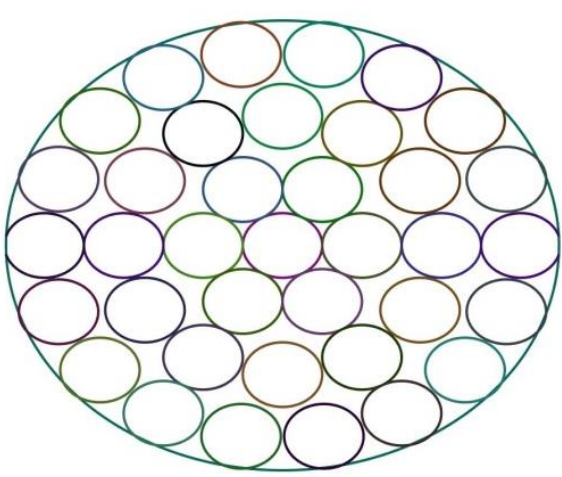

Fig.7 - Simulation result for $\mathbf{N}=37$

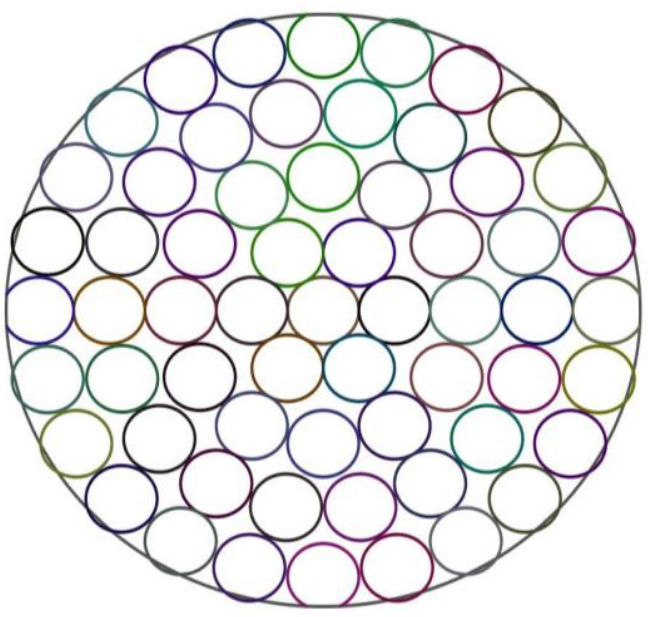

Fig.8 - Simulation result for $N=61$

It evident from Table I and II that CPU run time for ring packing is less whereas the packing efficiency of the hexagon packing is higher as it is the 'natural' shape. The reason for higher computation time in hexagon packing is the complexity in calculating the co-ordinates for the next location. Also, the area is maximum for a circle, hence hexagon packing in a sphere yields the best results possible in accordance with the mathematicians, computer scientists and physicists. 
Figure 3 and 6 shows comparison of CPU run time required by best known algorithms and hexagonal and ring packing for the 70 simulated cases. In best known results CPU run time increases exponential as the no of circles to be packed increases. Heuristics used here have shown minimum and consistent CPU run time irrespective of no of circles to be packed.

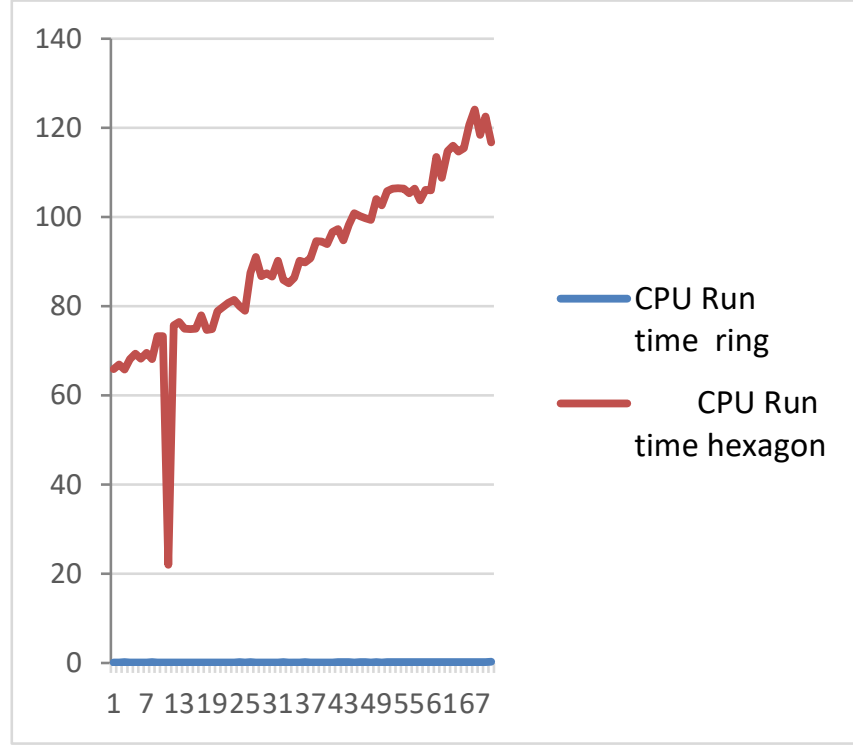

Fig.8 - Comparison of two packing methods

\section{CONCLUSION}

This paper solves the problem of packing unit circles into a larger containing circle using available modified basic heuristics rather than already available algorithms like ELP and Monte Carlo. The need for a suitable heuristic method is to ensure optimization of resources and revenue for implementing circle packing technique in the field of optical fibers, wireless sensor networks etc. A greedy method for obtaining heuristic solutions is thus modified and applied for the most relevant variant, occurring in a particular industrial setting. Whereas, the two sets of heuristics are competitive with the available literature and also comparable amongst each other for unit radius. In the future, we can remove the 'dead spaces' using the Monte Carlo Method and use meta-heuristics instead of a heuristic perspective. Socio Inspired optimization algorithms could be applied for handling the constraints.

\section{REFERENCES}

1. Liu, Jingfa, et al. "An improved energy landscape paving algorithm for the problem of packing circles into a larger containing circle." Computers \& Industrial Engineering 57.3 (2009): 1144-1149.

2. Wong, Wai Keung, et al. "Solving the two-dimensional irregular objects allocation problems by using a two-stage packing approach." Expert Systems with Applications 36.2 (2009): 3489-3496.

3. Li, S. P., and Ka-Lok Ng. "Study of the Unequal Spheres Packing Problem: An Application to Radiosurgery Treatment." International Journal of Modern Physics C 14.06 (2003): 815-823.

4. H. Poor, "An Introduction to Signal Detection and Estimation". New York: Springer-Verlag, 1985, ch. 4.

5. Hardin, Garrett. "The tragedy of the commons." science 162.3859 (1968): 1243-1248.

6. George, John A., Jennifer M. George, and Bruce W. Lamar. "Packing different-sized circles into a rectangular container." European Journal of Operational Research 84.3 (1995): 693-712.
7. Kubach, Timo, Andreas Bortfeldt, and Hermann Gehring. "Parallel greedy algorithms for packing unequal circles into a strip or a rectangle." Central European Journal of Operations Research 17.4 (2009): 461.

8. Kubach, Timo, et al. Parallel greedy algorithms for packing unequal spheres into a cuboidal strip or a cuboid. Fernuniv., Fachbereich Wirtschaftswiss., 2009.

9. Theodoracatos, Vassilios E., and James L. Grimsley. "The optimal packing of arbitrarily-shaped polygons using simulated annealing and polynomial-time cooling schedules." Computer methods in applied mechanics and engineering 125.1-4 (1995): 53-70.

10. Smith, A. P. "The sphere packing problem in quasicrystals." Journal of non-crystalline solids 153 (1993): 258-263. 